\title{
On the moments of the Carmichael $\lambda$ function
}

\author{
by \\ FLORIAN LUCA (Morelia) and \\ Ayyadurai Sankaranarayanan (Mumbai)
}

1. Introduction. Let $\lambda(n)$ be the universal exponent for the group of residues modulo $n$ that are coprime to $n$. The exact definition of this function is

$$
\lambda\left(p^{\nu}\right)= \begin{cases}p^{\nu-1}(p-1) & \text { if } p \geq 3 \text { or } \nu \leq 2, \\ 2^{\nu-2} & \text { if } p=2 \text { and } \nu \geq 3\end{cases}
$$

for a prime power $p^{\nu}$, and for an arbitrary integer $n \geq 2$,

$$
\lambda(n)=\operatorname{lcm}\left[\lambda\left(p_{1}^{\nu_{1}}\right), \ldots, \lambda\left(p_{k}^{\nu_{k}}\right)\right],
$$

where $n=p_{1}^{\nu_{1}} \cdots p_{k}^{\nu_{k}}$ is the prime factorization of $n$. Note that $\lambda(1)=1$.

A thorough analysis of the function $\lambda(n)$ was done in [3], where the minimal order, the normal order and the average value of this function were investigated. In particular, Theorem 3 in [3] shows that the estimate

$$
\frac{1}{x} \sum_{n \leq x} \lambda(n)=\frac{x}{\log x} \exp \left(\frac{B \log \log x}{\log \log \log x}(1+o(1))\right)
$$

holds as $x \rightarrow \infty$, where

$$
B=e^{-\gamma} \prod_{q}\left(1-\frac{1}{(q-1)^{2}(q+1)}\right)=0.34537 \ldots
$$

The above product is taken over prime values of $q$. In this paper, we use the method from [3] to estimate the higher moments of $\lambda(n)$. We have the following result.

Theorem 1. Let $r>0$ be fixed. Then the estimate

$$
M_{r}(x):=\frac{1}{x} \sum_{n \leq x} \lambda(n)^{r}=\frac{x^{r}}{\log x} \exp \left(\frac{B_{r} \log \log x}{\log \log \log x}(1+o(1))\right)
$$

2000 Mathematics Subject Classification: Primary 11N37; Secondary 11N13, 11N36. Key words and phrases: Carmichael $\lambda$ function, higher moments, smooth primes. 
holds as $x \rightarrow \infty$, where

$$
B_{r}=e^{-\gamma} \frac{2}{2^{r+1}-1} \prod_{q>2}\left(1-\frac{1}{(q-1)^{2}}\right)\left(1+\frac{q-1}{(q-2)\left(q^{r+1}-1\right)}\right) .
$$

It is important to note that the higher moments of the Carmichael $\lambda$ function (i.e., for large $r$ ) convey very strong information on the frequency of large values of the Carmichael $\lambda$ function.

We now turn our attention to negative values of $r$. It is much more difficult to estimate $M_{r}(x)$ for negative values of $r$. Studying such moments is important because they convey strong information on the frequency of small values of the Carmichael $\lambda$ function. While Theorem 1 shows that $M_{r}(x)=x^{r+o(1)}$ if $r>0$, this is no longer the case if $r<0$. In fact, when $r<0$, a trivial lower bound is $M_{r}(x) \geq x^{r}$. To be able to formulate our next result, we introduce some more notation. For a positive integer $n$, we write $P(n)$ for the largest prime factor of $n$. We have the following result.

Theorem 2. Assume that $\delta>0$ is a positive constant such that if we write

$$
\mathcal{P}_{\delta}(y)=\left\{p \leq y: P(p-1)<y^{\delta}\right\},
$$

then there exist constants $K=K(\delta)$ and $y_{0}(\delta)$ such that the inequality

$$
\# \mathcal{P}_{\delta}(y) \geq \frac{y}{(\log y)^{K}}
$$

holds for all $y>y_{0}(\delta)$. Then, for every fixed $r<0$, the lower bound

$$
M_{r}(x) \geq x^{-\delta+o(1)}
$$

holds as $x \rightarrow \infty$.

Our Theorem 2 shows that for some fixed value of $\delta \in(0,1)$, we have $M_{r}(x) \geq x^{-\delta}$ for large $x$, and independently of $r$. Of course, there is a gap in our result, in the sense that when $0>r>-\delta$, our result is worse than the trivial bound $M_{r}(x) \geq x^{r}$. According to Theorem 1 in [2], one can take $\delta=.2961$. It is conjectured that for each $\delta>0$, the lower bound (3) holds with any fixed $K>1$ for all $y>y_{0}(\delta, K)$. If this were so, then we would deduce by Theorem 2 that $M_{r}(x)=x^{o(1)}$ (note that the upper bound is obvious). Perhaps one can show unconditionally that $M_{r}(x)=x^{o(1)}$ for $r<0$, but we have failed to find such an argument. Our proof of Theorem 2 uses results on the distribution of primes $p$ with $p-1$ smooth (i.e., for which $P(p-1)$ is small relative to $p)$, much in the spirit of the construction of infinitely many Carmichael numbers by Alford, Granville and Pomerance from [1]; in fact, their construction shows that for large $x$ there are at least $x^{\beta}$ Carmichael numbers up to $x$, where $\beta=0.290306 \ldots$ Recall that a Carmichael number is a composite integer $n$ such that the congruence $a^{n} \equiv a$ 
$(\bmod n)$ holds for all integers $a$. More recently, Harman [4] adapted the above construction to produce more than $x^{0.33}$ Carmichael numbers up to $x$.

Throughout this paper, we use the Vinogradov symbols $\gg$ and $\ll$ and the Landau symbols $O$ and $o$ with their regular meaning. The constants implied by them might depend on the parameter $r$. We recall that $U \ll V$ and $V \gg U$ are both equivalent to the assertion that $U=O(V)$. For a positive real number $x$, we use $\log x$ for the maximum of the natural $\operatorname{logarithm}$ of $x$ (denoted $\ln x$ ) and 1 . We use $p$ and $q$ for prime numbers. We use $c_{0}, c_{1}, \ldots$ for positive constants which might depend on $r$ and which are labeled increasingly throughout the paper.

Acknowledgements. This paper was written during a very enjoyable visit of the first author at the Tata Institute of Fundamental Research in Fall of 2005 under an Associated Membership Scheme from the Third World Academy of Sciences. This author wishes to express his thanks to these institutions for the hospitality and support. The authors are thankful to the anonymous referee for some fruitful comments. The first author also thanks Professor William Banks for a useful conversation and for suggesting some of the questions addressed in this paper.

2. Proof of Theorem 1. We follow the method from [3]. Let $\mathcal{S}_{1}, \ldots, \mathcal{S}_{D}$ be disjoint sets whose union is the set of all odd primes less than or equal to $x$. Define

$$
E_{i}=\sum_{\substack{p^{\alpha} \leq x, \alpha \geq 1 \\ p \in \mathcal{S}_{i}}} \frac{1}{p^{\alpha}} .
$$

We write $\mathbf{j}$ for a vector $\left(j_{1}, \ldots, j_{D}\right)$ where each $j_{i}$ is a non-negative integer, and $\|\mathbf{j}\|=j_{1}+\cdots+j_{D}$. Finally, let $\mathcal{C}(x, \mathbf{j})$ be the set of integers $\leq x$ having exactly $j_{i}$ distinct prime divisors in $\mathcal{S}_{i}$. We put $y=\log \log x$. The following upper bound on $\mathcal{C}(x, \mathbf{j})$ appears in [3].

Proposition 1. There exists an absolute constant $c_{0}>0$ such that, for any $x>e^{e}$, and all vectors $\mathbf{j}$ as defined above, we have

$$
\# \mathcal{C}(x, \mathbf{j}) \leq \frac{c_{0} x}{(\log x)^{y}}+\frac{c_{0} x y}{\log x}\left(\prod_{i=1}^{D} \frac{E_{i}^{j_{i}}}{j_{i} !}\right)\left(\sum_{i=1}^{D} \frac{j_{i}}{E_{i}}\right) .
$$

(If $\mathcal{S}_{i}$ is empty, then we set $0 / E=0$ and $0^{0}=1$.)

We now specialize. We put $m=\left\lfloor y /(\log y)^{3}\right\rfloor$, let $D=m$ ! and define

$$
\mathcal{S}_{k}=\{p \leq x: \operatorname{gcd}(p-1, D)=2 k\} .
$$

The expressions $E_{i}$ for the above choices of the sets $\mathcal{S}_{i}$ were estimated in Lemma 1 in [3]. 
Lemma 1. Let $A>0$ be any fixed constant. For $k \leq(\log y)^{A}$, we have the following uniform asymptotic estimate:

$$
E_{k}=\frac{y P_{k}}{\log y}(1+o(1)), \quad \text { where } \quad P_{k}=\frac{e^{-\gamma}}{k} \prod_{q>2}\left(1-\frac{1}{(q-1)^{2}}\right) \prod_{q \mid k, q>2} \frac{q-1}{q-2} .
$$

Also, there is an absolute constant $c_{1}>0$ such that for all $2 k \mid D$, we have $E_{k}>1 / D^{c_{1}}$.

Actually, Lemma 1 above has been proved in [3] only for $A=2$, but a close analysis of that proof shows that this holds in the somewhat larger range as indicated above.

2.1. The upper bound. For a positive integer $n$, we write $\omega(n)$ for the number of distinct prime factors of $n$. Certainly,

$$
M_{r}(x)=\frac{1}{x} \sum_{n \leq x} \lambda(n)^{r}=\frac{1}{x} \sum_{\substack{n \leq x \\ \omega(n) \leq y^{2}}} \lambda(n)^{r}+\frac{1}{x} \sum_{\substack{n \leq x \\ \omega(n)>y^{2}}} \lambda(n)^{r} .
$$

The second sum on the right hand side of (4) is negligible because there are only $O\left(x /(\log x)^{2}\right)$ positive integers $n \leq x$ with more than $y^{2}$ prime factors. Indeed, to see this, one may either take $D=1$ in Proposition 1 , or sum up the well known inequality of Hardy and Ramanujan, namely

$$
\pi_{k}(x)=\#\{n \leq x: \omega(n)=k\} \ll \frac{x}{(k-1) !}(\log \log x+O(1))^{k}
$$

for all $k>y^{2}$.

The first sum on the right hand side of (4) equals

$$
S:=\frac{1}{x} \sum_{\|\mathbf{j}\|<y^{2}} \sum_{n \in \mathcal{C}(x, \mathbf{j})} \lambda(n)^{r} .
$$

For $n \in \mathcal{C}(x, \mathbf{j})$, we have

$$
\lambda(n)<\frac{D \phi(n)}{\prod_{k=1}^{D}(2 k)^{j_{k}}}<\frac{D x}{\prod_{k=1}^{D}(2 k)^{j_{k}}} .
$$

Combining the above estimate (5) with Proposition 1, we get the upper bound

$$
\begin{aligned}
S \leq & \frac{c_{0} x^{r} y D^{r}}{\log x} \sum_{\|\mathbf{j}\|<y^{2}}\left(\prod_{k=1}^{D} \frac{E_{k}^{j_{k}}}{(2 k)^{r j_{k} j_{k} !}}\right)\left(\sum_{k=1}^{D} \frac{j_{k}}{E_{k}}\right) \\
& +\frac{c_{0} x^{r} D^{r}}{(\log x)^{\log y}} \sum_{\|\mathbf{j}\|<y^{2}} \prod_{k=1}^{D} \frac{1}{(2 k)^{r j_{k}}} .
\end{aligned}
$$


To estimate the second sum, note that

$$
\sum_{\|\mathbf{j}\|<y^{2}} \prod_{k=1}^{D} \frac{1}{(2 k)^{r j_{k}}}=\prod_{k=1}^{D} \frac{1}{1-(1 / 2 k)^{r}}<c_{2}(2 D)^{r},
$$

where

$$
c_{2}=\prod_{k=1}^{m_{r}} \frac{1}{1-(1 / 2 k)^{r}}
$$

and $m_{r}$ is the smallest positive integer such that the inequality

$$
\max \left\{1,(2 n)^{r}\right\}<(2(n+1))^{r}-1
$$

holds for all $n \geq m_{r}$. Thus,

$$
\frac{c_{0} x^{r} D^{r}}{(\log x)^{\log y}} \sum_{\|\mathbf{j}\|<y^{2}} \prod_{k=1}^{D} \frac{1}{(2 k)^{r j_{k}}} \ll \frac{x^{r}(2 D)^{2 r}}{(\log x)^{\log y}},
$$

and since

$$
(2 D)^{2 r}=(2 m !)^{2 r}=\exp (O(r m \log m))=\exp \left(O\left(r y /(\log y)^{2}\right)\right),
$$

while $(\log x)^{\log y}=\exp (y \log y)$, we see that this sum is negligible even uniformly for $r<c_{3} \log y$, for some appropriate absolute constant $c_{3}$. Thus, the second term in estimate (6) is negligible. For the first, note that since $\|\mathbf{j}\|<y^{2}$, we have, by Lemma 1 , that

$$
\sum_{k=1}^{D} \frac{j_{k}}{E_{k}}<\frac{y^{2} D}{D^{c_{1}}}
$$

Hence, we need only to estimate

$$
\left(\frac{x^{r} y^{3} D^{r+1-c_{1}}}{\log x}\right) \sum_{\|\mathbf{j}\|<y^{2}}\left(\prod_{k=1}^{D} \frac{E_{k}^{j_{k}}}{(2 k)^{r j_{k}} j_{k} !}\right),
$$

which is certainly less than

$$
\begin{aligned}
&\left(\frac{x^{r} y^{3} D^{r+1-c_{1}}}{\log x}\right) \exp \left(\sum_{k=1}^{D} \frac{E_{k}}{(2 k)^{r}}\right) \\
&=\left(\frac{x^{r}}{\log x}\right) \exp \left(\sum_{k=1}^{D} \frac{E_{k}}{(2 k)^{r}}+o\left(\frac{y}{\log y}\right)\right),
\end{aligned}
$$

since by our choice of $D$, we have

$$
\begin{aligned}
D^{1+r-c_{1}} & =\exp \left(\left(1+r-c_{1}\right) \log (m !)\right) \\
& \leq \exp \left(\left(1+r-c_{1}\right) m \log m\right)=\exp \left(o\left(\frac{y}{\log y}\right)\right),
\end{aligned}
$$

even uniformly in $r=o(\log y)$. 
For the sum in the exponent in (7), we split it at $\ell=\left\lfloor(\log y)^{2 / r}+1\right\rfloor$, getting

$$
\sum_{k=1}^{D} \frac{E_{k}}{(2 k)^{r}}=\sum_{k=1}^{\ell} \frac{E_{k}}{(2 k)^{r}}+\sum_{k=\ell+1}^{D} \frac{E_{k}}{(2 k)^{r}} .
$$

We now show that the second sum is negligible. Indeed, by the BrunTitchmarsh inequality, it is easy to see that $E_{k} \ll y / \phi(k)$. Thus, using partial summation and the estimate

$$
\sum_{k \leq u} \frac{1}{\phi(k)} \ll \log u
$$

which holds uniformly in $u>1$, we obtain

$$
\sum_{k=\ell+1}^{D} \frac{E_{k}}{(2 k)^{r}} \ll \frac{1}{2^{r}} \sum_{k=\ell+1}^{D} \frac{y}{k^{r} \phi(k)} \ll \frac{1}{r 2^{r}} \frac{y}{\ell^{r}} \ll \frac{y}{2^{r} r(\log y)^{2}}=o\left(\frac{y}{\log y}\right),
$$

even uniformly when $1 / r=o(\log y)$. Finally, by Lemma 1 with $A=2 / r$ for a fixed $r$ (or, at least, for some $r$ which remains bounded away from zero), we find that the first $\operatorname{sum} \sum_{k=1}^{\ell} E_{k} /(2 k)^{r}$ is asymptotic to

$$
\begin{array}{r}
\frac{y}{\log y} e^{-\gamma} \prod_{q>2}\left(1-\frac{1}{(q-1)^{2}}\right) \sum_{k=1}^{\ell}\left(\frac{1}{2^{r} k^{r+1}} \prod_{q \mid k, q>2} \frac{q-1}{q-2}\right) \\
=B_{r}(1+o(1)) \frac{y}{\log y}
\end{array}
$$

where

$$
B_{r}=\frac{e^{-\gamma}}{2^{r}} \prod_{q>2}\left(1-\frac{1}{(q-1)^{2}}\right) \sum_{k=1}^{\infty} \frac{1}{k^{r+1}} \prod_{q \mid k, q>2} \frac{q-1}{q-2} .
$$

Observing that the function

$$
\frac{1}{k^{r+1}} \prod_{q \mid k, q>2} \frac{q-1}{q-2}
$$

is multiplicative, we can further simplify the expression for $B_{r}$ to

$$
\begin{aligned}
B_{r}= & \frac{e^{-\gamma}}{2^{r}}\left(1+\frac{1}{2^{r+1}}+\frac{1}{2^{2(r+1)}}+\cdots\right) \prod_{q>2}\left(1-\frac{1}{(q-1)^{2}}\right) \\
& \times\left(1+\frac{1}{q^{r+1}} \frac{q-1}{q-2}+\frac{1}{q^{2(r+1)}} \frac{q-1}{q-2}+\cdots\right),
\end{aligned}
$$

which leads to the expression (2) after grouping the geometric series. This proves the upper bound in Theorem 1. 
2.2. The lower bound. For the proof of the lower bound, we define:

- $\Omega_{1}(x ; \mathbf{j})$ as the set of positive integers $n$ that can be formed by picking $v=\|\mathbf{j}\|$ distinct primes $p_{1}, \ldots, p_{v}$ in such a way that

(a) $p_{i}<x^{1 / y^{3}}$ for all $i$,

(b) the first $j_{1}$ primes are in $\mathcal{S}_{1}$, the next $j_{2}$ in $\mathcal{S}_{2}$, etc.;

- $\Omega_{2}(x ; \mathbf{j})$ consists of those integers $s=p_{1} \cdots p_{v} \in \Omega_{1}(x, \mathbf{j})$ with the additional property that $\operatorname{gcd}\left(p_{i}-1, p_{j}-1\right)$ divides $D$ for all $i \neq j$;

- $\Omega_{3}(x ; \mathbf{j})$ consists of all integers of the form $n=s p$, where $s \in \Omega_{2}(x ; \mathbf{j})$ and $p \in \mathcal{S}_{1}$ satisfies $\max \left(x /(2 s), x^{1 / y}\right)<p \leq x / s$;

- $\Omega_{4}(x ; \mathbf{j})$ consists of all integers $n=\left(p_{1} \cdots p_{v}\right) p \in \Omega_{3}(x ; \mathbf{j})$ with the additional property that $\operatorname{gcd}\left(p-1, p_{i}-1\right)=2$ for all $i=1, \ldots, v$.

Now we can proceed with the proof of the lower bound. Throughout this proof, we will use Lemmas $2-4$ from [3]. We put $\ell_{1}=\lfloor\log y\rfloor$ and let $J$ be the set of all j's with $0 \leq j_{k} \leq E_{k} /\left(2^{r-1} k^{r}\right)$ for $k \leq \ell_{1}$, and $j_{k}=0$ for $k>\ell_{1}$. Evidently

$$
M_{r}(x)=\frac{1}{x} \sum_{n \leq x} \lambda(n)^{r} \geq \frac{1}{x} \sum_{\mathbf{j} \in J} \sum_{n \in \Omega_{4}(x ; \mathbf{j})} \lambda(n)^{r} .
$$

Lemma 2 in [3] yields the lower bound (with $j_{k}=0$ for $k>\ell_{1}$ )

$$
\frac{1}{x} \sum_{\mathbf{j} \in J} \sum_{n \in \Omega_{4}(x ; \mathbf{j})} \lambda(n)^{r} \geq\left(\frac{c_{3}}{y}\right)^{r} x^{r-1} \sum_{\mathbf{j} \in J} \prod_{k=1}^{\ell_{1}}(2 k)^{-r j_{k}} \sum_{n \in \Omega_{4}(x ; \mathbf{j})} 1 .
$$

To estimate the innermost sum, note that

$$
\sum_{n \in \Omega_{4}(x ; \mathbf{j})} 1=\sum_{s \in \Omega_{2}(x ; \mathbf{j})} \sum_{\left\{p:(s p) \in \Omega_{4}(x ; \mathbf{j})\right\}} 1 .
$$

By Lemma 3 on p. 380 of [3], the above sum is greater than

$$
\frac{c_{4} x}{y \log x} \sum_{s \in \Omega_{2}(x ; \mathbf{j})} \frac{1}{s} .
$$

Checking the fact that the hypotheses of that lemma are fulfilled amounts to proving that $\|\mathbf{j}\| \leq y^{2}$, but for $\mathbf{j} \in J$, we have, by Lemma 1 ,

$$
\begin{aligned}
\|\mathbf{j}\| & \leq \frac{1}{2^{r-1}} \sum_{k \leq \ell_{1}} \frac{E_{k}}{k^{r}} \ll \frac{y}{\log y} \sum_{k \geq 1} \frac{1}{k^{1+r}} \\
& =\zeta(1+r) \frac{y}{\log y} \ll\left(1+\frac{1}{r}\right) \frac{y}{\log y} ;
\end{aligned}
$$


hence, the inequality $\|\mathbf{j}\|<y^{2}$ holds for large $x$. Thus, estimates (8)-(11) imply

$$
M_{r}(x) \geq \frac{c_{3}^{r} c_{4} x^{r}}{y^{r+1} \log x} \sum_{\mathbf{j} \in J} \prod_{k=1}^{\ell_{1}}(2 k)^{-r j_{k}} \sum_{s \in \Omega_{2}(x ; \mathbf{j})} \frac{1}{s} .
$$

Lemma 4 in [3] shows that this sum is greater than

$$
\begin{aligned}
& \frac{x^{r}}{\log x} \exp \left(\frac{-c_{5} y}{(\log y)(\log \log y)^{2}}+O(r \log y)\right) \sum_{\mathbf{j} \in J} \prod_{k=1}^{\ell_{1}} \frac{E_{k}^{j_{k}}}{(2 k)^{r j_{k}} j_{k} !} \\
&=\frac{x^{r}}{\log x} \exp \left(o\left(\frac{y}{\log y}\right)\right) \prod_{k=1}^{\ell_{1}} \sum_{k=0}^{\left\lfloor E_{k} /\left(2^{r-1} k^{r}\right)\right\rfloor} \frac{\left(E_{k} /(2 k)^{r}\right)^{j_{k}}}{j_{k} !} .
\end{aligned}
$$

Finally, since

$$
\sum_{j=0}^{\lfloor 2 w\rfloor} \frac{w^{j}}{j !}>\frac{e^{w}}{2} \quad \text { for all } w \geq 1
$$

we get

$$
\begin{aligned}
M_{r}(x) & \geq \frac{x^{r}}{2^{\ell_{1}} \log x} \exp \left(\sum_{k=1}^{\ell_{1}} \frac{E_{k}}{(2 k)^{r}}+o\left(\frac{y}{\log y}\right)\right) \\
& =\frac{x^{r}}{\log x} \exp \left(\frac{B_{r} y}{\log y}+o\left(\frac{y}{\log y}\right)\right) .
\end{aligned}
$$

3. Proof of Theorem 2. We assume that $\delta<1$. We let $y$ be a large positive real number. For simplicity, we write $\mathcal{P}$ for the set $\mathcal{P}_{\delta}(y)$. We let $\mathcal{P}_{1}$ be the subset of $p \in \mathcal{P}$ such that $p>y /(\log y)^{2 K}$ and $p-1$ is not divisible by any powerful number $a>z=(\log y)^{4 K}$. Recall that $a$ is powerful if $q^{2} \mid a$ for every prime factor $q$ of $a$. Since the counting function of the set of powerful numbers $\leq t$ is $O\left(t^{1 / 2}\right)$, it follows easily that the number of $n \leq y$ admitting a powerful divisor $a>z$ is

$$
\leq \sum_{\substack{z<a \leq y \\ a \text { powerful }}} \frac{y}{a} \ll \frac{y}{\sqrt{z}}=\frac{y}{(\log y)^{2 K}} .
$$

We thus deduce that the estimate

$$
\# \mathcal{P}_{1} \geq \# \mathcal{P}+O\left(\frac{y}{(\log y)^{2 K}}\right)>\frac{y}{2(\log y)^{K}}
$$

holds for large $y$.

For any subset $\mathcal{S}$ of $\mathcal{P}_{1}$, let $m_{\mathcal{S}}$ be the square-free positive integer whose prime factors are precisely the elements of $\mathcal{S}$. Note that, by unique factorization, different subsets $\mathcal{S} \subset \mathcal{P}_{1}$ lead to distinct values of the positive 
integer $m_{\mathcal{S}}$. Let $N=\left\lfloor y^{\delta}\right\rfloor$, and let

$$
\mathcal{R}=\left\{m_{\mathcal{S}}: \mathcal{S} \subset \mathcal{P}_{1} \text { and } \# \mathcal{S}=N\right\}
$$

We first find a lower bound for the number of elements of $\mathcal{R}$. Since $\delta<1$, it follows that

$$
2 N<2 y^{\delta}<\frac{y}{2(\log y)^{K}}<\# \mathcal{P}_{1}
$$

therefore

$$
\# \mathcal{R}=\left(\begin{array}{c}
\# \mathcal{P}_{1} \\
N
\end{array}\right) \geq\left(\frac{\# \mathcal{P}_{1}}{N}\right)^{N}=\exp (N(1-\delta+o(1)) \log y)
$$

Further, if $m \in \mathcal{R}$, then

$$
m<y^{N}=\exp (N \log y) .
$$

We write $x:=\exp \left(y^{\delta} \log y\right)$. We now bound $\lambda(m)$ for $m \in \mathcal{R}$. Clearly,

$$
\lambda(m)=\operatorname{lcm}[p-1: p \mid m] .
$$

Assume that $q^{\alpha} \| \lambda(m)$, where $q$ is prime. Then $q \leq y^{\delta}$ because every prime factor $p$ of $m$ is in $\mathcal{P}_{1}$. Further, since there exists $p \mid m$ such that $q^{\alpha} \mid(p-1)$, we see that if $\alpha>1$, then $q^{\alpha}<z$, therefore

$$
\alpha \leq c_{6} \log z=4 c_{6} K \log \log y,
$$

where $c_{6}=1 / \ln 2$. Hence,

$$
\lambda(n) \leq\left(\prod_{q \leq y^{\delta}} q\right)^{4 c_{6} K \log \log y}=\exp \left(O\left(y^{\delta} \log \log y\right)\right),
$$

where in the last inequality above we used the Prime Number Theorem. Thus,

$$
\begin{aligned}
M_{r}(x) & \geq x^{-1} \sum_{m \in \mathcal{R}} \lambda(n)^{r} \\
& \geq x^{-1} \# \mathcal{R} \min \left\{\lambda(n)^{r}: n \in \mathcal{R}\right\} \\
& =\exp (-(N+O(1)) \log y+(1-\delta) N \log y+O(r N \log \log y)) \\
& =\exp (-(\delta+o(1)) N \log y)=x^{-\delta+o(1)},
\end{aligned}
$$

which completes the proof of Theorem 2 .

\section{References}

[1] W. R. Alford, A. Granville and C. Pomerance, There are infinitely many Carmichael numbers, Ann. of Math. (2) 139 (1994), 703-722.

[2] R. C. Baker and G. Harman, Shifted primes without large prime factors, Acta Arith. 83 (1998), 331-361.

[3] P. Erdős, C. Pomerance and E. Schmutz, Carmichael's lambda function, ibid. 58 (1991), 363-385. 
[4] G. Harman, On the number of Carmichael numbers up to $x$, Bull. London Math. Soc. 37 (2005), 641-650.

Instituto de Matemáticas

Universidad Nacional Autónoma de México

C.P. 58089

Morelia, Michoacán, México

E-mail: fluca@matmor.unam.mx
School of Mathematics Tata Institute of Fundamental Research Homi Bhabha Road Mumbai 400 005, India E-mail: sank@math.tifr.res.in

Received on 3.12.2005

and in revised form on 1.3.2006 